\title{
LIPA GENE MUTATIONS AFFECT THE COMPOSITION OF LIPOPROTEINS: ENRICHMENT IN ACAT-DERIVED CHOLESTERYL ESTERS
}

Lorenzo Arnaboldi $^{\mathrm{a}, 1}$, Alice Ossoli ${ }^{\mathrm{b}, 1}$, Eleonora Giorgio ${ }^{\mathrm{b}}$, Livia Pisciotta ${ }^{\mathrm{c}, \mathrm{d}}$, Tiziano Lucchi ${ }^{\mathrm{e}}$, Liliana Grigore ${ }^{\dagger}$, Chiara Pavanello ${ }^{b}$, Agnese Granata $^{a}$, Andrea Pasta ${ }^{c}$, Beatrice Arosio ${ }^{e, g}$, Domenico Azzolino ${ }^{\mathrm{e}, \mathrm{g}}$, Andrea Baragetti ${ }^{\mathrm{a}}$, Samuela Castelnuovo ${ }^{\mathrm{h}}$, Alberto Corsini $\mathrm{i}^{\mathrm{a}, \mathrm{i}}$, Alberico L. Catapano ${ }^{\mathrm{a}, \mathrm{i}}$, Laura Calabresi ${ }^{\mathrm{b}}$ and Monica Gomaraschi ${ }^{\mathrm{b},{ }^{*}}$

a Dipartimento di Scienze Farmacologiche e Biomolecolari, Università degli Studi di Milano,

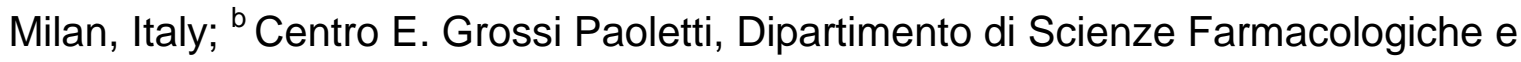
Biomolecolari, Università degli Studi di Milano, Milan, Italy; ${ }^{\mathrm{c}}$ Department of Internal Medicine, University of Genova, Italy; ${ }^{\mathrm{d}}$ Policlinic Hospital San Martino, Genoa, Italy; ${ }^{\mathrm{e}}$ Metabolic Disease Clinic - Geriatric Unit, Fondazione IRCCS Ca' Granda Ospedale Maggiore Policlinico, Milan, Italy; ${ }^{\dagger}$ S.I.S.A. Center for the Study of Atherosclerosis, Bassini Hospital, Cinisello Balsamo, Italy; ${ }^{9}$ Department of Clinical Sciences and Community Health, University of Milan, Italy; ${ }^{\text {h }}$ Centro Dislipidemie, ASST Ospedale Niguarda, Milan, Italy; ' IRCCS Multimedica Hospital, Milan, Italy.

${ }^{1}$ co-first authors

* Corresponding author:

Monica Gomaraschi, PhD

Centro E. Grossi Paoletti, Dipartimento di Scienze Farmacologiche e Biomolecolari Università degli Studi di Milano

Via Balzaretti 9, 20133 Milano, Italy

E-mail: monica.gomaraschi@unimi.it

Word count: 3865

Tables and figures: 1 table and 4 figures.

Keywords: Cholesteryl ester storage disease; Lysosomal acid lipase; Lipoproteins. 


\section{ABSTRACT}

Background and aims

Cholesteryl ester storage disease (CESD) due to LIPA gene mutations is characterized by hepatic steatosis, hypercholesterolemia and hypoalphalipoproteinemia, exposing affected patients to an increased cardiovascular risk. Further insights into the impact of LIPA gene mutations on lipid/lipoprotein metabolism are limited. Aim of the study was to investigate the effect of carrying one or two mutant LIPA alleles on lipoprotein composition and function. Methods

Lipoproteins were isolated from 6 adult CESD patients, 5 relatives carrying one mutant LIPA allele (carriers) and 12 sex/age matched controls. Lipid profile, lipoprotein mass composition and the fatty acid distribution of cholesteryl esters (CEs) were assessed. HDL function was evaluated as the ability to promote nitric oxide release by endothelial cells.

Results

Despite the lipid-lowering therapy, total cholesterol, LDL-cholesterol and triglycerides were increased in CESD patients compared to controls, while HDL-cholesterol was reduced.

Carriers also displayed elevated total and LDL-cholesterol. Very low and intermediate density lipoproteins from CESD patients and carriers were enriched in CEs compared to the control ones, with a concomitant reduction of triglycerides. Fatty acid composition of CEs in serum and lipoproteins showed a depletion of linoleate content in CESD patients, due to the reduced LCAT activity. In CESD HDL, fatty acid distribution of CEs was shifted towards saturated ones, if compared to control HDL. The changes in HDL composition did not affect HDL ability to promote nitric oxide release by endothelial cells.

Conclusions

LIPA gene mutations significantly affected plasma levels and lipid composition of lipoproteins, likely contributing to the increased cardiovascular risk of affected patients. 


\section{Introduction}

Lysosomal acid lipase (LAL) is a key regulator of cellular lipid homeostasis; it catalyzes the hydrolysis of cholesteryl esters (CEs) and triglycerides (TGs), which are taken up through the receptor-mediated endocytosis of apoB-containing lipoproteins, in the lysosomal compartment [1].

Unesterified cholesterol (UC) and free fatty acids (FFAs) generated by LAL are then released in the cytosol, where they can regulate their own synthesis and metabolism. LAL is coded by the LIPA gene on chromosome 10, and mutations affecting enzyme expression or function cause two recessive autosomal diseases depending on residual LAL activity: Wolman disease (WD) and Cholesteryl Ester Storage Disease (CESD) [2]. WD is the most severe form of LAL deficiency (LAL-D) with a LAL activity below $1 \%$ than normal and results in demise in the first year of life for hepatic and adrenal failure. CESD is the later-onset type that may present in infancy, childhood or adulthood, with residual LAL activity within $1 \%$ and $12 \%$ than normal values. Clinically, CESD patients present with hepatomegaly, splenomegaly, malabsorption and increased cardiovascular risk [3]. Consistently, carotid intima-media thickness (CIMT), a marker of preclinical atherosclerosis, is elevated in CESD patients; however, a direct comparison with age- and sex-matched controls is lacking $[4,5]$. Biochemically, hypercholesterolemia (variably associated with hypertriglyceridemia) and low plasma levels of HDL-cholesterol (HDL-C) have been described, together with elevation of liver enzymes [3]. Few studies analyzed carriers of one mutant LIPA allele; even in the absence of a clinical phenotype, some alterations of the lipid profile were detected in heterozygotes if compared to non carriers [6,7].

Clinical and biochemical features of LAL-D are the consequence of the accumulation of CEs and, to a lesser extent, TGs in the lysosomes. Since LAL is ubiquitously expressed, lipid accumulation occurs in all tissues, but especially in the liver and in macrophages throughout the body [3]. Lipid accumulation in the liver causes a peculiar microvescicular steatosis [8,9], which can evolve to fibrosis and cirrhosis. Interestingly, the rate of progression of liver disease in CESD patients is higher than that of other chronic liver diseases, as NAFLD or hepatitis C; indeed, the median time to the first documentation of fibrosis, cirrhosis, or liver transplantation was estimated as 3.1 years from the first clinical manifestation of LAL-D [10]. In hepatocytes, the lack of UC and FFA release in the cytosol results in the SREBP-mediated upregulation of cholesterol and fatty acid synthesis, leading to increased VLDL secretion and 
hypercholesterolemia [11-13]. The complex alterations of lipid metabolism induced by LAL-D, including the inhibition of ABCA1 expression with a reduced HDL biogenesis, also lead to the low plasma levels of HDL-C observed in CESD patients [14].

Further insights into the impact of genetic LAL-D on the structural and functional features of the different lipoprotein classes are limited. In particular, since CEs play a central role in the pathology of LAL-D, it would be relevant to known whether the content of CEs is altered in CESD lipoproteins; in addition, understanding which esterification system is mainly responsible for $\mathrm{CE}$ synthesis could provide the rationale for the development of novel therapeutic strategies for LAL-D. The esterification of cholesterol can occur intracellularly or within the plasma compartment. Acyl-coenzyme A:cholesterol acyltransferase (ACAT) is located in the endoplasmic reticulum and catalyzes the synthesis of CEs in the cytosol by conjugating cholesterol to long-chain fatty acids, mainly oleic and palmitic acids [15]. On the contrary, lecithin:cholesterol acyltransferase (LCAT) is the only enzyme responsible for CE generation in plasma; it acts on cholesterol carried by all lipoprotein classes and unsaturated FAs are its preferred substrates, especially linoleate [16]. Unfortunately, being ACAT an intracellular enzyme, its expression and activity cannot be easily evaluated in blood samples, as for LCAT; however, it is possible to infer ACAT and LCAT contribution to CE generation by evaluating CE fatty acid composition [17-19].

Regarding the impact of genetic LAL-D on lipoprotein function, we previously showed that cholesterol efflux capacity of HDL is impaired in CESD patients [20]. It is well known that atheroprotection by HDL is also mediated by several activities that are independent from their role in cholesterol transport, as the ability to preserve endothelial homeostasis [21]. In this context, the ability of HDL to promote the release of nitric oxide (NO) from endothelial cells is a widely used and reproducible assay of HDL function [22]. No evidence of the impact of genetic LAL-D on endothelial protection by HDL is available. 


\section{Materials and Methods}

\subsection{Patients, Carriers and Controls}

Blood samples were collected from 6 CESD patients. Cases 1 and 2 were twins carrying the c.894 G>A (p.S275_Q298) and c.652 C>T (p.R218X) LIPA gene mutations. Case 3 was compound heterozygote for the c.894 G>A (p.S275_Q298) and the c.883C>T (p.H295Y) mutations. Case 4 was compound heterozygote for the c.652 C>T (p.R218X) and c.881 T>C (p.L294S) mutations. Case 5 was homozygote for the c.894 G>A (p.S275_Q298) mutation. All CESD patients presented with hypercholesterolemia, elevated transaminases and hepatic steatosis (Supplemental table 1) [4,23-25]. Case 6 was homozygote for the c.894 G>A (p.S275_Q298) mutation and heterozygote for the c.137T>C (p.L46P) mutation in the $A P O E$ gene [26]; interestingly, she displayed hypercholesterolemia with low HDL-C, but normal transaminases and no sign of hepatic steatosis (Supplemental table 1). All cases were on lipid-lowering therapy; none was treated with enzyme replacement therapy at the time of analysis (Supplemental table 1).

Five carriers of one mutant LIPA allele, hereafter referred to as "carriers", were enrolled among cases' parents $(n=4)$ or during the screening for the $c .894 \mathrm{G}>A$ mutation $(n=1)$. One subject carried the c.652 C>T (p.R218X) mutation and four subjects carried the c.894 G>A (p.S275_Q298) LIPA gene mutation. Two carriers were on statin therapy.

A group of healthy subjects, matched for sex and age at a 2:1 ratio to CESD patients, was enrolled.

Carotid IMT measurements were performed by high-resolution B-mode carotid ultrasonography as previously described [27]. Briefly, the far walls of the left and right common carotids, bifurcations, and internal carotids were visualized in anterior, lateral, and posterior projections. Images were saved as JPEG files on a digital support. Carotid IMT measurements were performed using a dedicated software that allows semiautomatic edge detection of the echogenic lines of the intima-media complex (M'Ath, Metris S.R.L., France). All carotid measurements were averaged to calculate the mean IMT for each subject. The study conformed to the guidelines set out in the Declaration of Helsinki and was approved by the ethical committee of Milano Area C (n. 582-112015); all enrolled patients gave written informed consent for participation in the study.

\subsection{Biochemical analyses}


Blood samples were collected after an overnight fast. Plasma levels of total cholesterol (TC), TGs, HDL-C, apolipoprotein A-I and B, aspartate aminotransferase, alanine aminotransferase, and gamma glutamyltransferase were measured by certified enzymatic and immunoturbidimetric assays on a c311 automatic analyzer (Roche Diagnostics). LDLcholesterol (LDL-C) was calculated by the Friedewald formula.

LCAT activity was measured as the ability of endogenous LCAT to esterify cholesterol incorporated into an exogenous standardized substrate [28]. The substrate was a reconstituted HDL (rHDL) made of apoA-I, palmitoyloleylphosphatidylcholine and cholesterol at a weight ratio of $1: 2.17: 0.11$ (corresponding to a molar ratio of $1: 80: 8$ ), prepared by the cholate dialysis technique [29]. Plasma and rHDL were mixed at a 1:10 volume ratio and incubated for $1 \mathrm{~h}$ at $37^{\circ} \mathrm{C}$. UC was measured before and after the incubation by a standard enzymatic assay in the absence of cholesterol esterase. Absorbance at $510 \mathrm{~nm}$ was measured with a Synergy $\mathrm{H} 1$ multi-mode reader (BioTek).

LAL activity on dried blood spots (DBS) was measured by fluorescence using 4methylumbelliferone palmitate (Cayman Chemicals), cardiolipin (Avanti Polar Lipids) and the selective LAL inhibitor Lalistat 2 (kindly provided by Alexion Pharma), according to the method of Hamilton et al. [30]. The generation of fluorescent 4-methylumbelliferone (4-MU) was detected by the Synergy H1 Multi-Mode microplate reader and GEN5 software (BioTek). LAL activity was calculated by subtracting the activity in the inhibited reaction (with Lalistat 2) from uninhibited reaction (with $\mathrm{H}_{2} \mathrm{O}$ ) and expressed as nmols of generated 4-MU/spot/h. Normal values of LAL activity are $>0.80 \mathrm{nmol} / \mathrm{spot} / \mathrm{h}$.

\subsection{Lipoprotein isolation and composition}

VLDL (d<1.006 g/ml), IDL (d=1.006-1.020 g/ml), LDL (d=1.020-1.063 g/ml) and HDL $(\mathrm{d}=1.063-1.21 \mathrm{~g} / \mathrm{ml})$ were isolated by sequential ultracentrifugation from the plasma of CESD patients, carriers and controls. TC, UC, TG and phospholipid (PL) content of the isolated lipoproteins was measured by standard enzymatic techniques; the CE mass was calculated as $(T C-U C) \times 1.68$. Protein content was assessed by the method of Lowry. Lipoprotein composition was expressed as absolute values and as percentage of particle total mass. For each lipoprotein, total mass was calculated as the sum of protein, PL, TG, UC and CE concentrations. 


\subsection{Cholesteryl ester fatty acid composition}

Serum and lipoprotein samples underwent multiple extractions at $4^{\circ} \mathrm{C}$ with chloroform/methanol 2:1, butylated hydroxytoluene (BHT) $0.01 \%$ as antioxidant, and $\mathrm{KCl}$ $0.05 \%$. The pooled organic phases were dried under a stream of nitrogen and resuspended in chloroform/methanol 2:1 with BHT. Aliquots were loaded onto a TLC developed in hexane:diethylether:acetic acid 80:20:1. After run, TLCs were sprayed with dichlorofluorescein and the spots corresponding to CEs were removed. CEs underwent derivatization with methanolic $\mathrm{HCl} 3 \mathrm{~N}$ for 120 minutes at $80^{\circ} \mathrm{C}$, and extraction by hexane/water. CE fatty acid (CEFA) content was analyzed by a DANI 1000 GLC (Dani, Milano, Italy) equipped with a flame ionization detector and a HTA autosampler (HTA, Brescia, Italy) [31]. FA peaks were identified by comparing their retention times with a standard mixture (FAME MIX 37, Sigma-Aldrich) and the area under the curve (AUC) was determined by automated integration with a dedicated software (Clarity, Prague, Czech Republic). Fatty acid composition of CEs is reported as percentage of total AUC. Only for sera, TG spots were also removed after TLC and their fatty acid composition was assessed as described above.

\subsection{NO production in endothelial cells}

Sera from CESD patients, carriers and controls were incubated with $20 \%$ polyethylene glycol for 20 minutes to precipitate apoB-containing lipoproteins [32]. ApoB-depleted (apoB-D) sera were tested for their ability to promote NO production in human umbilical vein endothelial cells (HUVECs, PromoCell, Carlo Erba Reagents). NO production was evaluated as previously described [33]. Briefly, HUVECs were incubated with 5\% (v/v) apoB-D sera for 30 minutes and $\mathrm{NO}$ generation was detected by fluorescence using diacetate 4,5-diaminofluorescein (DAF-2 DA, Sigma-Aldrich Chemie). Fluorescence intensity was measured with a Synergy $\mathrm{H} 1$ Multi-Mode microplate reader equipped with the GEN5 software (BioTek). For each sample, fluorescence was normalized by the protein concentration of the total cell lysate. To address the equivalence between apoB-D sera and isolated HDL as inducers of NO, HUVECs were incubated with apoB-D sera or isolated HDL from controls and NO generation was assessed as described above.

\subsection{Statistical analysis}


Data are expressed as mean $\pm S D$, if not otherwise stated. Comparisons between groups were performed by one-way ANOVA for independent samples. Non-normally distributed variables were log-transformed before proceeding to the analysis. Tests were two-sided and $p$ values $<0.05$ were considered as statistically significant. Differences in carotid IMT between CESD patients and controls were assessed by covariance analysis (ANCOVA) and adjusted for age, sex and plasma lipids. NO production by HDL or by apoB-D sera were compared by Pearson correlation. Statistical analysis was performed using SPSS version 24.0 software (SPSS Inc., Chicago, USA). 


\section{Results}

3.1 Biochemical and clinical features of CESD patients, carriers and controls CESD patients, carriers and controls were comparable for gender distribution and age on average (Table 1). LAL activity was almost undetectable in CESD patients; carriers also displayed a significant reduction of LAL activity, well below the lower reference limit of 0.8 $\mathrm{nmol} / \mathrm{spot} / \mathrm{h}$ (Table 1). Controls displayed normal LAL activity values.

Despite the lipid-lowering therapy, plasma levels of TC, LDL-C, TG and apoB were significantly elevated in CESD patients if compared to controls (Table 1). Interestingly, carriers also displayed higher plasma levels of TC, LDL-C and apoB than controls, even when adjusted for age and sex. HDL-C and apoA-I levels were reduced only in CESD patients. As expected, plasma levels of liver transaminases were significantly higher in CESD patients than in carriers and controls. Overall, a gene-dose-dependent increase of LDL-C, TG, apoB, alanine aminotransferase and gamma glutamyltransferase was detected.

Mean carotid IMT tended to be higher in CESD patients compared to controls $(0.75 \pm 0.19$ vs $0.61 \pm 0.13 \mathrm{~mm}, p=0.054)$, and the difference became significant after adjustment for sex and age (Fig. 1, $p_{\text {ANCOVA }}=0.005$ ). Further adjustment for TC, LDL-C or TG did not affect the relationship between IMT and LIPA genotype ( $p_{\mathrm{ANCOVA}}=0.008,0.009$ and 0.006 , respectively); on the contrary, the difference was lost after adjustment for HDL-C $\left(p_{\text {ANCOVA }}=0.223\right)$.

\subsection{Lipoprotein composition}

Absolute and percentage lipoprotein compositions are reported in Supplementary table 2 and Figure 2, respectively. VLDL and IDL percentage compositions were significantly affected by the presence of LIPA gene mutations (Fig. 2). VLDL from CESD patients showed a higher content of CEs than control VLDL $(13.8 \pm 1.9 \%$ vs $6.7 \pm 3.3 \%$, respectively $p<0.001)$, with a concomitant reduction of TG content $(53.3 \pm 4.4 \%$ vs $63.1 \pm 5.2 \%, p=0.002)$. PL content was also increased from $13.4 \pm 3.0 \%$ in control VLDL to $18.7 \pm 3.9 \%(p=0.006)$ in CESD VLDL. These changes were even more evident in IDL: CEs increased from $17.2 \pm 6.7 \%$ in control IDL to $28.7 \pm 4.1 \%$ in CESD IDL ( $p=0.002$ ). In addition, TGs were almost halved in CESD IDL $(21.3 \pm 7.8 \%$ vs $39.5 \pm 7.5 \%$ in control IDL, $p=0.019)$. PL content was also significantly higher in CESD IDL compared to the control ones ( $23.5 \pm 5.4 \%$ vs $16.4 \pm 4.0 \%$, respectively). Interestingly, the percentage compositions of VLDL and IDL from carriers of one mutant LIPA allele were almost identical to those of CESD lipoproteins. Consistently, the absolute content 
of CEs in VLDL and IDL was significantly higher in CESD patients and carriers compared to controls (Supplementary table 2); here, the concomitant higher concentrations of proteins and PLs suggest an increased number of circulating VLDL and IDL particles in CESD patients. The percentage compositions of LDL and HDL did not show major changes among CESD patients, carriers and controls (Fig. 2).

\subsection{CEFA composition of plasma and lipoprotein fractions}

The fatty acid composition of CEs accumulating in the lipoproteins of CESD patients was then assessed (Supplemental Table 3). A tendency towards a reduction of CEs containing 18:2 with a mild increase of those containing 18:1 was observed in sera from CESD patients (Fig. 3). To exclude a possible role of the diet, TG fatty acid composition was also assessed; no significant changes were detected between CESD patients, carriers and controls (Supplemental Table 4).

Even if serum CEFA profile was mainly representative of the LDL composition, a significant reduction in the percentage content of 18:2 was also observed in CESD VLDL and HDL, again with a gene-dose-dependent trend (Fig. 3). When compared to lipoproteins from controls, 18:2 content was 8.9\% lower in VLDL, 7.4\% lower in LDL and 12.5\% lower in HDL isolated from CESD patients. CESD HDL also displayed a significantly higher content of 16:0 than control HDL (16.9 $\pm 2.6 \%$ vs $12.3 \pm 1.5 \%$, respectively) (Fig. 3). Consequently, in CESD HDL the percentage distribution of CEs containing saturated, monounsaturated or polyunsaturated fatty acids was shifted towards the saturated ones, if compared to control HDL profile (Fig. 4A). No significant changes were detected in VLDL and LDL (data not shown).

The reduced content of 18:2 affected the CE oleate/linoleate ratio in the three main lipoprotein classes: a gene-dose dependent increase of the 18:1/18:2 ratio was observed in lipoproteins from subjects carrying mutations in the LIPA gene when compared to control ones, consistent with what observed on whole sera (Supplemental table 5). The reduced 18:2 content of CE and the higher oleate/linoleate ratio observed in carriers of LIPA gene mutations were likely due to a reduced cholesterol esterification by LCAT. Indeed, LCAT activity was significantly lower in CESD patients compared to controls, with a mean reduction of $38.0 \pm 9.3 \%$ (Supplementary figure 1). 


\subsection{NO production in HUVECs}

Since the CEFA composition of HDL was significantly affected by LIPA gene mutations, the impact of this alteration on HDL ability to promote NO production in endothelial cells was tested. The increased content of CEs carrying saturated fatty acid residues did not affect NO production by HDL; indeed, similar NO levels were detected in cells incubated with apoB-D sera from CESD patients, carriers and controls (Fig. 4B).

HDL ability to promote NO production was assessed using apoB-D sera added to endothelial cells at $5 \%(\mathrm{v} / \mathrm{v})$. Indeed, the incubation of HUVECs with isolated HDL or with apoB-D sera at the same final HDL-C concentration induced a comparable increase of NO production (Supplemental Figure 2). The equivalence between isolated HDL and apoB-D serum was further tested in a wide range of concentrations. When individual apoB-D sera were added at a final volume between $2 \%$ and $30 \%$, NO generation reached a maximum at $5 \%$ and decreased at higher concentrations; at 30\%, apoB-D sera had no effect on NO generation (Supplemental Figure 3). Again, no differences were detected between apoB-D sera and isolated $\mathrm{HDL}$ in the whole concentration range. When all the data were analyzed together, a significant positive correlation was found between the two methods $(R=0.794, p<0.001$, Supplemental Figure 4). 


\section{Discussion}

Cholesteryl ester storage disease is associated with hypercholesterolemia, hypoalphalipoproteinemia and possibly hypertriglyceridemia [3], indicating that circulating levels of pro-atherogenic lipoproteins are increased, while atheroprotective HDL are reduced. Thus, CESD patients are exposed to an increased cardiovascular risk. Indeed, we showed that CESD patients had higher carotid IMT values, index of pre-clinical atherosclerosis, than controls. Consistently, although in the absence of a direct comparison with matched controls, carotid IMT values of CESD patients were found to be $>75^{\text {th }}$ percentile of the general population with similar age and sex $[4,5]$.

We extended previous observations on genetic LAL-D by characterizing its impact on lipoproteins composition not only in CESD patients, but also in carriers of one mutant LIPA allele belonging to the same families. We showed that the number of circulating apoBcontaining lipoproteins is likely increased, consistent with the higher hepatic secretion of VLDL in LAL-D [13]; in addition, lipoprotein composition is altered. In particular, the core of VLDL is enriched in CEs and depleted in TGs. This alteration is even more evident in IDL. The reduced TG content of VLDL and IDL could be the consequence of an altered composition of VLDL during their hepatic assembly, coupled with a preserved TG hydrolysis by lipoprotein lipase. Interestingly, the composition of VLDL and IDL is similarly modified in heterozygous carriers, thus indicating that a $~ 50 \%$ inhibition of LAL enzymatic activity is enough to affect lipoprotein composition. These results imply that the higher content of CEs in apoB-containing lipoproteins could contribute to the lipid accumulation in the arterial wall and to the accelerated development of atherosclerosis observed in CESD patients. Then, the fatty acid composition of CEs was assessed with the aim to investigate their origin. Indeed, CEs are produced intracellularly by ACAT and included into nascent VLDL, or are generated in the plasma compartment by LCAT. The latter hypothesis was not supported by the significant reduction of linoleate (18:2), the preferred LCAT substrate [16], and of LCAT activity in CESD patients. Interestingly, the values of LCAT activity detected in CESD patients were similar to those of heterozygous carriers of LCAT mutations [34] and to those of patients with chronic kidney disease, in which an acquired LCAT deficiency was previously described [35]. The cause of LCAT impairment in LAL-D are presently unknown, but it could be the consequence of a defective LCAT production by the liver, a key target organ of LAL-D pathology. Consequently, it is possible to speculate on a relevant role of ACAT in the generation of CEs 

hepatic and intestinal accumulation of CEs in animal models of lysosomal storage disorders, as LAL-D and Niemann-Pick $C$ disease $[36,37]$. Our results suggest a possible additional role of ACAT in LAL-D pathology by affecting lipoprotein composition and atherosclerosis development, which is worth of confirmation in dedicated studies. The reduction of linoleate content in CEs was also evident in the other lipoprotein classes, as LDL and HDL; consequently, CE linoleate content was reduced in CESD sera, leading to a gene-dosedependent increase of the oleate/linoleate ratio. Although limited by the small sample size, our results improve the current knowledge on how a reduced LAL activity (and the consequent cellular compensatory mechanisms) can affect the lipid/lipoprotein profile of LAL$D$ patients. Even if TG fatty acid composition was similar between cases and controls, the impact of individual dietary habits on CEFA composition cannot be excluded, due to the lack of controls belonging to the same families and of a detailed nutritional assessment of enrolled subjects.

The impact of genetic LAL-D on HDL was investigated to extend our previous observations in a smaller group of pediatric CESD patients [20]. CESD HDL had a higher content of CEs containing saturated fatty acids in place of those containing polyunsaturated ones. In addition, HDL mass composition was characterized by a slight decrease of CEs and by a higher protein content, in line with our previous findings of smaller HDL in CESD patients [20]. Thus, genetic LAL-D not only impairs HDL biogenesis by the liver [14], but also affects HDL composition and subclass distribution. To address the impact of these changes on HDLmediated atheroprotection, their ability to promote nitric oxide release by endothelial cells was investigated, as a key process in endothelial homeostasis [21]. The results showed that CESD HDL fully retained their ability to stimulate nitric oxide release by endothelial cells in vitro. On the contrary, we previously showed that HDL ability to promote cell cholesterol efflux, the first step of the reverse transport of cholesterol from peripheral tissue to the liver [38], is impaired in CESD patients, with an impact on all the tested efflux pathways [20]. This partial impairment of HDL function could contribute to the increased cardiovascular risk of CESD patients. Consistently, the difference in carotid IMT between CESD patients and controls was lost after adjustment for HDL-C.

The characterization of the lipid/lipoprotein profile in genetic LAL-D was extended to carriers of one mutant LIPA allele. Consistent with previous findings $[6,7]$, the lipid profile of these 
carriers is altered if compared to controls; indeed, a gene-dose-dependent effect of LAL-D was observed on plasma LDL-C levels and liver enzymes, but not on HDL-C. Together with the altered composition of carriers' lipoproteins, these findings indicate that even partial LAL$D$ can affect plasma lipid/lipoprotein profile. Thus, even if LAL-D is classified as a recessive disease and heterozygosity for LIPA gene mutations was not associated to an increased risk of cardiovascular events [39], further studies are needed to assess whether carrying one mutant LIPA allele could predispose to hepatic and/or atherosclerotic disease in the presence of other risk factors.

\section{Conflict of interest}

AC received honoraria from AstraZeneca, AMGEN, Sanofi, Recordati, Novartis, MSD, Mediolanum, DOC, Mylan and Pfizer. ALC received honoraria, lecture fees, or research grants from Akcea, Amgen, Astrazeneca, Eli Lilly, Genzyme, Kowa, Mediolanum, Menarini, Merck, Pfizer, Recordati, Sanofi, Sigma Tau, Medco and Amryt. LC received grants from Alexion Pharma, Medlmmune and Daiichi Sankyo. MG received grants from Alexion Pharma.

\section{Financial support}

This work was partially supported by an intramural grant for young researchers of Università degli Studi di Milano to MG (Linea 2, azione A).

\section{Author contribution}

LA and AO: execution of the experiments, manuscript preparation. $E G$ and $A G$ : execution of the experiments. CP: data management and analysis. LP, TL, LG, AP, BA, DA, AB, SC: identification, clinical management and characterization of patients and controls, provision of biological material. AC, ALC and LC: interpretation of the findings and review of the manuscript. MG: study design, data analysis and interpretation, manuscript preparation. 


\section{References}

[1] N.K. Desai, D.P. Wilson, Lysosomal Acid Lipase Deficiency, In: Feingold KR, et al, Endotext, South Dartmouth (MA), (2000).

[2] Grabowski GA, Chamas L, Du H, Lysosomal Acid Lipase Deficiencies: The Wolman Disease/Cholesteryl Ester Storage Disease Spectrum. In: The Metabolic and Molecular Bases of Inherited Disease, McGraw-Hill, 2012.

[3] D.L. Bernstein, H. Hulkova, M.G. Bialer, R.J. Desnick, Cholesteryl ester storage disease: Review of the findings in 135 reported patients with an underdiagnosed disease, J.Hepatol. 58 (2013) 1230-1243.

[4] L. Pisciotta, G. Tozzi, L. Travaglini, R. Taurisano, T. Lucchi, et al, Molecular and clinical characterization of a series of patients with childhood-onset lysosomal acid lipase deficiency. Retrospective investigations, follow-up and detection of two novel LIPA pathogenic variants, Atherosclerosis 265 (2017) 124-132.

[5] P. Poinsot, F.S. Collardeau, L. Restier, A. Serusclat, M. Di Filippo, et al, Childhood/adult-onset lysosomal acid lipase deficiency: A serious metabolic and vascular phenotype beyond liver disease-four new pediatric cases, J.Clin.Lipidol. 11 (2017) 167-177.

[6] S. Muntoni, H. Wiebusch, M. Jansen-Rust, S. Rust, H. Schulte, et al, Heterozygosity for lysosomal acid lipase E8SJM mutation and serum lipid concentrations, Nutr.Metab Cardiovasc.Dis. 23 (2013) 732-736.

[7] C.R. Pullinger, E.O. Stock, I. Movsesyan, M.J. Malloy, P.H. Frost, et al, Identification and metabolic profiling of patients with lysosomal acid lipase deficiency, J.Clin.Lipidol. 9 (2015) 716-726.

[8] V. Ivashkin, M. Zharkova, Cholesteryl Ester Crystals in Lysosomal Acid Lipase Deficiency, N.Engl.J.Med. 376 (2017) e14.

[9] H. Hulkova, M. Elleder, Distinctive histopathological features that support a diagnosis of cholesterol ester storage disease in liver biopsy specimens, Histopathology 60 (2012) 1107-1113.

[10] B.K. Burton, N. Silliman, S. Marulkar, Progression of liver disease in children and adults with lysosomal acid lipase deficiency, Curr.Med.Res.Opin. 33 (2017) 1211-1214.

[11] J.L. Goldstein, S.E. Dana, J.R. Faust, A.L. Beaudet, M.S. Brown, Role of lysosomal acid lipase in the metabolism of plasma low density lipoprotein. Observations in cultured fibroblasts from a patient with cholesteryl ester storage disease, J.Biol.Chem. 250 (1975) 8487-8495.

[12] H.N. Ginsberg, N.A. Le, M.P. Short, R. Ramakrishnan, R.J. Desnick, Suppression of apolipoprotein B production during treatment of cholesteryl ester storage disease with 
lovastatin. Implications for regulation of apolipoprotein B synthesis, J.Clin.Invest. 80 (1987) 1692-1697.

[13] M.H. Cummings, G.F. Watts, Increased hepatic secretion of very-low-density lipoprotein apolipoprotein B-100 in cholesteryl ester storage disease, Clin.Chem. 41 (1995) 111-114.

[14] K.L. Bowden, N.J. Bilbey, L.M. Bilawchuk, E. Boadu, R. Sidhu, et al, Lysosomal Acid Lipase Deficiency Impairs Regulation of ABCA1 Gene and Formation of High Density Lipoproteins in Cholesteryl Ester Storage Disease, J.Biol.Chem. 286 (2011) 3062430635.

[15] L.L. Rudel, R.G. Lee, P. Parini, ACAT2 is a target for treatment of coronary heart disease associated with hypercholesterolemia, Arterioscler.Thromb.Vasc.Biol. 25 (2005) 1112-1118.

[16] L. Calabresi, S. Simonelli, M. Gomaraschi, G. Franceschini, Genetic lecithin:cholesterol acyltransferase deficiency and cardiovascular disease, Atherosclerosis 222 (2012) 299-306.

[17] T.P. Carr, J.S. Parks, L.L. Rudel, Hepatic ACAT activity in African green monkeys is highly correlated to plasma LDL cholesteryl ester enrichment and coronary artery atherosclerosis, Arterioscler.Thromb. 12 (1992) 1274-1283.

[18] J.W. Furbee, Jr., O. Francone, J.S. Parks, In vivo contribution of LCAT to apolipoprotein $B$ lipoprotein cholesteryl esters in LDL receptor and apolipoprotein $\mathrm{E}$ knockout mice, J.Lipid Res. 43 (2002) 428-437.

[19] R.G. Lee, K.L. Kelley, J.K. Sawyer, R.V. Farese, Jr., J.S. Parks, et al, Plasma cholesteryl esters provided by lecithin:cholesterol acyltransferase and acyl-coenzyme a:cholesterol acyltransferase 2 have opposite atherosclerotic potential, Circ.Res. 95 (2004) 998-1004.

[20] F. Zimetti, E. Favari, P. Cagliero, M.P. Adorni, N. Ronda, et al, Cholesterol traffickingrelated serum lipoprotein functions in children with cholesteryl ester storage disease, Atherosclerosis 242 (2015) 443-449.

[21] L. Calabresi, M. Gomaraschi, G. Franceschini, Endothelial protection by high-density lipoproteins: from bench to bedside, Arterioscler.Thromb.Vasc.Biol. 23 (2003) 17241731.

[22] F. O'Neill, E. McLoughlin, M. Riwanto, J. Manz, A. Adler, et al, Reproducibility and biological variability of HDL's vascular functional assays, Atherosclerosis 241 (2015) 588-594.

[23] R. Longhi, C. Vergani, R. Valsasina, E. Riva, C. Galluzzo, et al, Cholesteryl ester storage disease: risk factors for atherosclerosis in a 15-year-old boy, J.Inherit.Metab Dis. 11 Suppl 2 (1988) 143-145. 
[24] S. Decarlis, C. Agostoni, F. Ferrante, S. Scarlino, E. Riva, et al, Combined hyperlipidaemia as a presenting sign of cholesteryl ester storage disease, J.Inherit.Metab Dis. 32 Suppl 1 (2009) S11-S13.

[25] T. Fasano, L. Pisciotta, L. Bocchi, O. Guardamagna, P. Assandro, et al, Lysosomal lipase deficiency: molecular characterization of eleven patients with Wolman or cholesteryl ester storage disease, Mol.Genet.Metab 105 (2012) 450-456.

[26] M. Orth, W. Weng, H. Funke, A. Steinmetz, G. Assmann, et al, Effects of a frequent apolipoprotein $\mathrm{E}$ isoform, ApoE4Freiburg (Leu28-- >Pro), on lipoproteins and the prevalence of coronary artery disease in whites, Arterioscler.Thromb.Vasc.Biol. 19 (1999) 1306-1315.

[27] L. Calabresi, D. Baldassarre, S. Simonelli, M. Gomaraschi, M. Amato, et al, Plasma lecithin:cholesterol acyltransferase and carotid intima-media thickness in European individuals at high cardiovascular risk, J.Lipid Res. 52 (2011) 1569-1574.

[28] L. Calabresi, L. Pisciotta, A. Costantin, I. Frigerio, I. Eberini, et al, The molecular basis of lecithin:cholesterol acyltransferase deficiency syndromes: a comprehensive study of molecular and biochemical findings in 13 unrelated Italian families, Arterioscler Thromb Vasc Biol 25 (2005) 1972-1978.

[29] A. Jonas, J.H. Wald, K.L. Toohill, E.S. Krul, K.E. Kezdy, Apolipoprotein A-I structure and lipid properties in homogeneous, reconstituted spherical and discoidal high density lipoproteins, J.Biol.Chem. 265 (1990) 22123-22129.

[30] J. Hamilton, I. Jones, R. Srivastava, P. Galloway, A new method for the measurement of lysosomal acid lipase in dried blood spots using the inhibitor Lalistat 2, Clin.Chim.Acta 413 (2012) 1207-1210.

[31] L. Da Dalt, M. Ruscica, F. Bonacina, G. Balzarotti, A. Dhyani, et al, PCSK9 deficiency reduces insulin secretion and promotes glucose intolerance: the role of the low-density lipoprotein receptor, Eur.Heart J. 40 (2019) 357-368.

[32] B.F. Asztalos, M. de la Llera-Moya, G.E. Dallal, K.V. Horvath, E.J. Schaefer, et al, Differential effects of HDL subpopulations on cellular ABCA1- and SR-BI-mediated cholesterol efflux, J.Lipid Res. 46 (2005) 2246-2253.

[33] M. Gomaraschi, A. Ossoli, E. Favari, M.P. Adorni, G. Sinagra, et al, Inflammation impairs eNOS activation by HDL in patients with acute coronary syndrome, Cardiovasc.Res. 100 (2013) 36-43.

[34] L. Calabresi, E. Favari, E. Moleri, M.P. Adorni, M. Pedrelli, et al, Functional LCAT is not required for macrophage cholesterol efflux to human serum, Atherosclerosis 204 (2009) 141-146.

[35] L. Calabresi, S. Simonelli, P. Conca, G. Busnach, M. Cabibbe, et al, Acquired lecithin:cholesterol acyltransferase deficiency as a major factor in lowering plasma HDL levels in chronic kidney disease, J.Intern.Med. 277 (2015) 552-561. 
[36] A.M. Lopez, J.C. Chuang, K.S. Posey, T. Ohshiro, H. Tomoda, et al, PRD125, a potent and selective inhibitor of sterol O-acyltransferase 2 markedly reduces hepatic cholesteryl ester accumulation and improves liver function in lysosomal acid lipasedeficient mice, J.Pharmacol.Exp.Ther. 355 (2015) 159-167.

[37] A.M. Lopez, R.D. Jones, J.J. Repa, S.D. Turley, Niemann-Pick C1-deficient mice lacking sterol O-acyltransferase 2 have less hepatic cholesterol entrapment and improved liver function, Am.J.Physiol Gastrointest.Liver Physiol 315 (2018) G454G463.

[38] M. Cuchel, D.J. Rader, Macrophage reverse cholesterol transport: key to the regression of atherosclerosis?, Circulation 113 (2006) 2548-2555.

[39] N.O. Stitziel, S.W. Fouchier, B. Sjouke, G.M. Peloso, A.M. Moscoso, et al, Exome sequencing and directed clinical phenotyping diagnose cholesterol ester storage disease presenting as autosomal recessive hypercholesterolemia, Arterioscler.Thromb.Vasc.Biol. 33 (2013) 2909-2914. 
FIGURE LEGENDS

Fig. 1. Mean carotid intima-media thickness (IMT) in CESD and controls. Values were adjusted for age and sex. Data are mean \pm SEM, $n=6$ for CESD and 12 for controls. ${ }^{*} p<0.05$ by ANCOVA.

Fig. 2. Lipoprotein composition. Mass percentage composition of lipoproteins isolated by ultracentrifugation from the plasma of CESD patients, carriers and controls. Data are expressed as mean $\pm S D, n=6$ for CESD, 5 for carriers and 12 for controls. ${ }^{*} p<0.05$ vs controls. PL, phospholipids; TG, triglycerides; UC, unesterified cholesterol; CE, cholesteryl esters.

Fig. 3. CEFA composition. Percentage composition of fatty acids in CEs from serum and lipoproteins of CESD patients, carriers and controls. Data are expressed as mean $\pm S D, n=6$ for CESD, 5 for carriers and 12 for controls. ${ }^{*} p<0.05$ vs controls. 16:0, palmitate; $18: 1$, oleate; 18:2, linoleate.

Fig. 4. HDL composition and function. (A) Percentage distribution of saturated (SFA), monounsaturated (MUFA) and polyunsaturated (PUFA) fatty acids in the CEs of HDL from CESD, carriers and controls. (B) NO production in HUVECs. Endothelial cells were incubated with $5 \%(\mathrm{v} / \mathrm{v})$ apoB-depleted sera from CESD patients, carriers and controls to promote NO production. Results are expressed as fold of increase in treated vs untreated cells. Data are expressed as mean $\pm S D, n=6$ for CESD, 5 for carriers and 12 for controls. ${ }^{*} p<0.05$ vs controls. 


\section{Table 1}

Biochemical features of CESD patients, carriers and controls

\begin{tabular}{|c|c|c|c|c|}
\hline & CESD & Carriers & Controls & $p$ \\
\hline$N(m / f)$ & $6(1 / 5)$ & $5(2 / 3)$ & $12(2 / 10)$ & 0.535 \\
\hline Age, y & $37.7 \pm 21.2$ & $58.6 \pm 17.9$ & $36.5 \pm 18.1$ & 0.149 \\
\hline LAL activity, nmol/spot/h & $0.01 \pm 0.01^{\star \#}$ & $0.57 \pm 0.26^{*}$ & $1.35 \pm 0.37$ & $<0.001$ \\
\hline Total cholesterol, mg/dl & $173.5 \pm 33.8^{*}$ & $182.0 \pm 32.3^{*}$ & $145.8 \pm 11.7$ & 0.015 \\
\hline Triglycerides, mg/dl & $105.3 \pm 47.0^{*}$ & $81.3 \pm 16.4$ & $57.0 \pm 14.1$ & 0.006 \\
\hline LDL-cholesterol, mg/dl & $121.5 \pm 26.7^{*}$ & $105.2 \pm 29.1^{*}$ & $80.0 \pm 15.5$ & 0.003 \\
\hline HDL-cholesterol, mg/dl & $31.0 \pm 10.1^{\star \#}$ & $62.0 \pm 10.9$ & $56.5 \pm 14.8$ & 0.001 \\
\hline Apolipoprotein B, mg/dl & $111.8 \pm 22.9^{\star \#}$ & $92.2 \pm 19.2^{*}$ & $66.1 \pm 7.5$ & $<0.001$ \\
\hline Apolipoprotein A-I, mg/dl & $87.2 \pm 17.0^{\star \#}$ & $130.2 \pm 13.6$ & $127.5 \pm 23.0$ & 0.001 \\
\hline Alanine aminotransferase, U/I & $64.2 \pm 31.2^{\star \#}$ & $18.0 \pm 7.5$ & $8.9 \pm 4.9$ & $<0.001$ \\
\hline Aspartate aminotransferase, $\mathrm{U} / \mathrm{I}$ & $48.8 \pm 11.5^{\star \#}$ & $19.1 \pm 3.9$ & $21.6 \pm 5.0$ & $<0.001$ \\
\hline Gamma glutamyltransferase, U/I & $40.1 \pm 33.0^{\star \#}$ & $17.9 \pm 6.0$ & $10.1 \pm 2.0$ & 0.007 \\
\hline
\end{tabular}




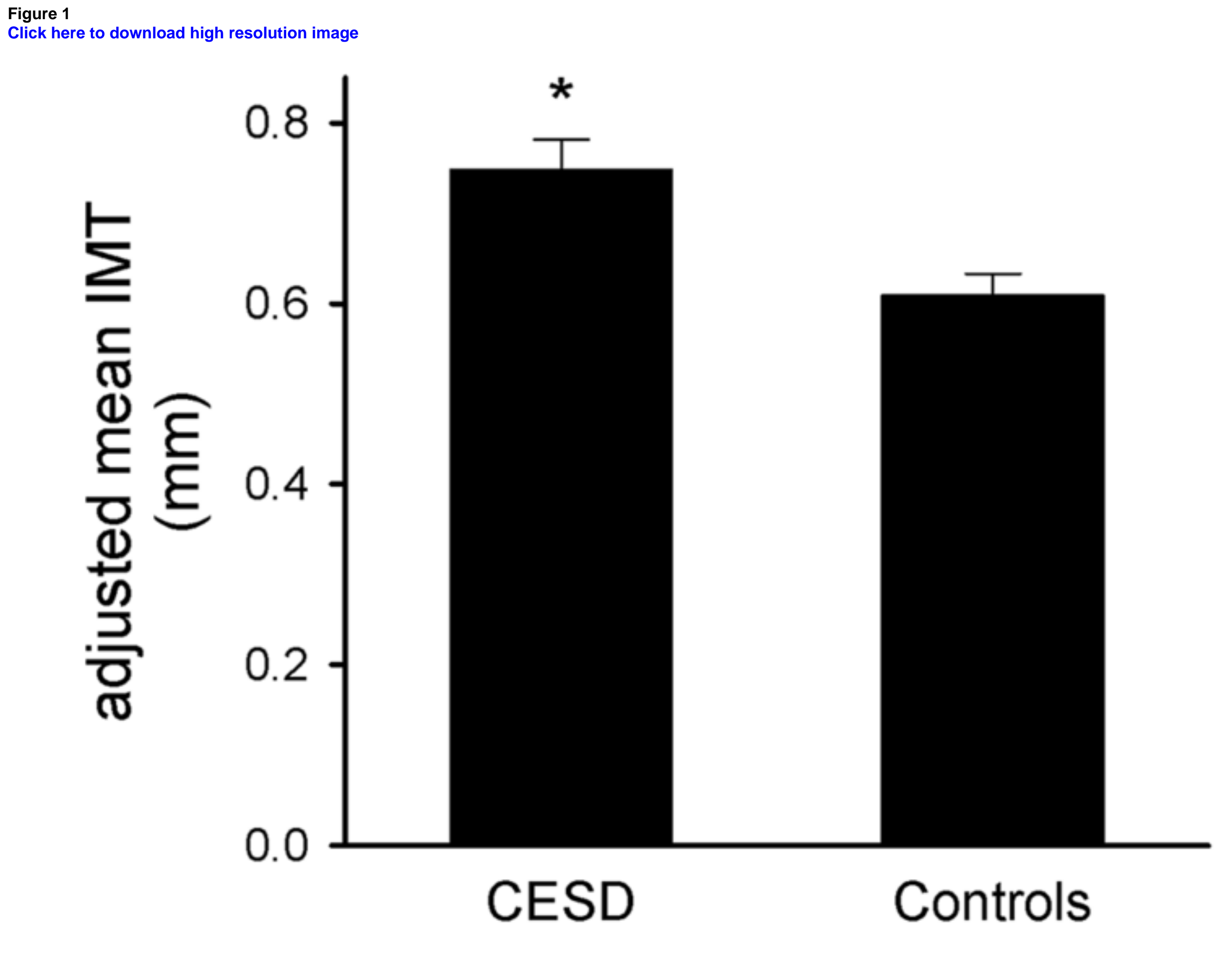

$0.8-*$

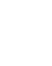



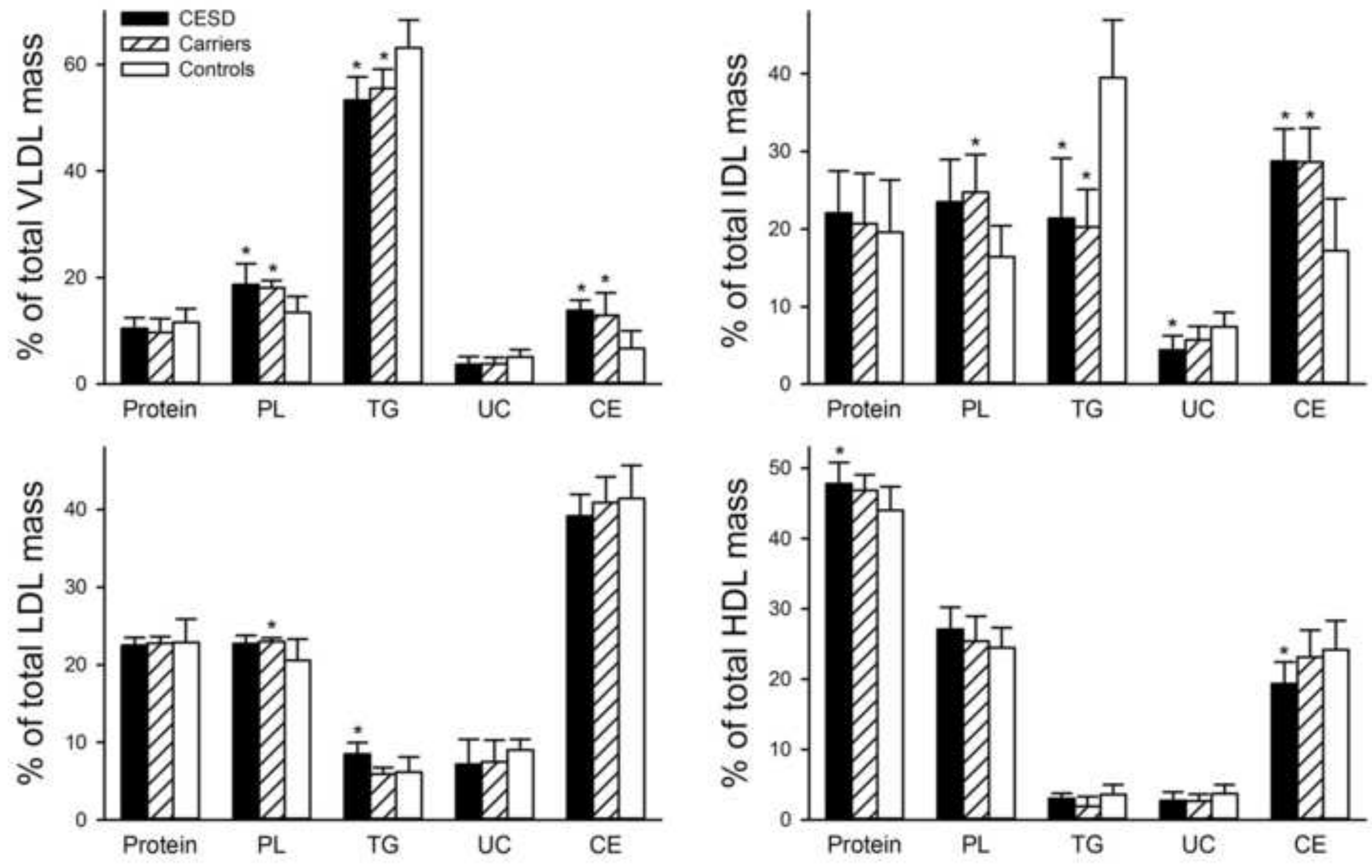

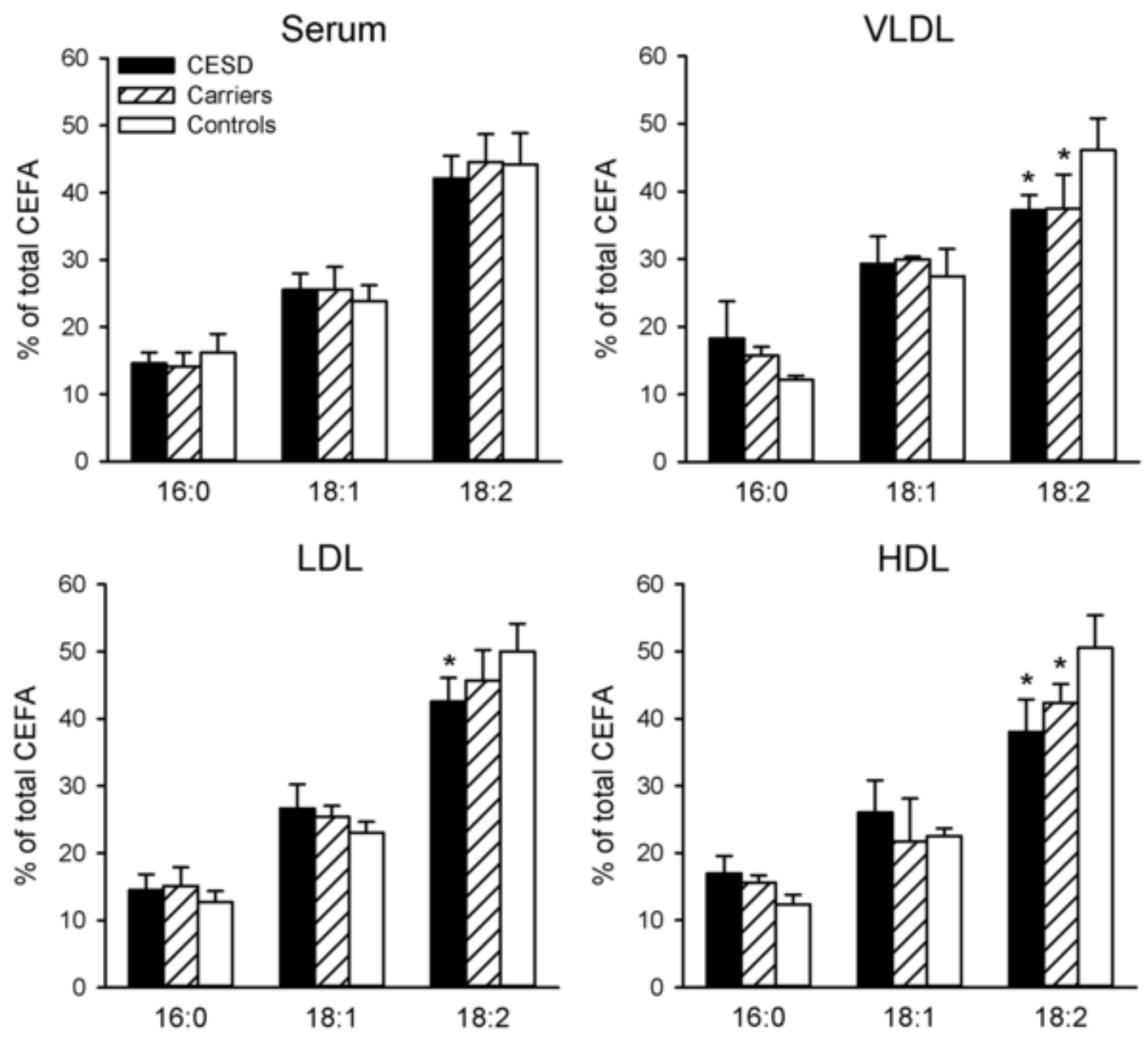
(A)

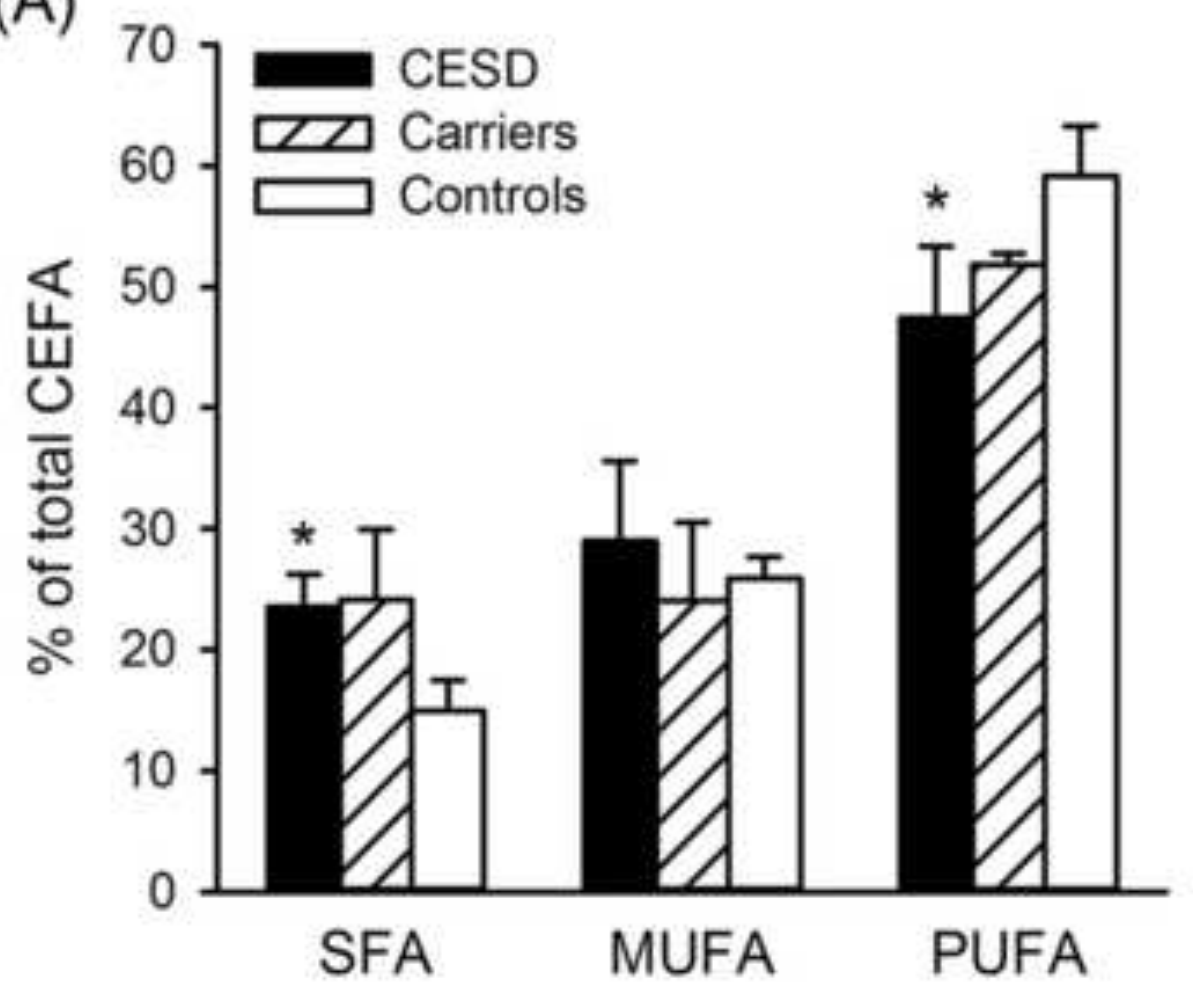

(B)

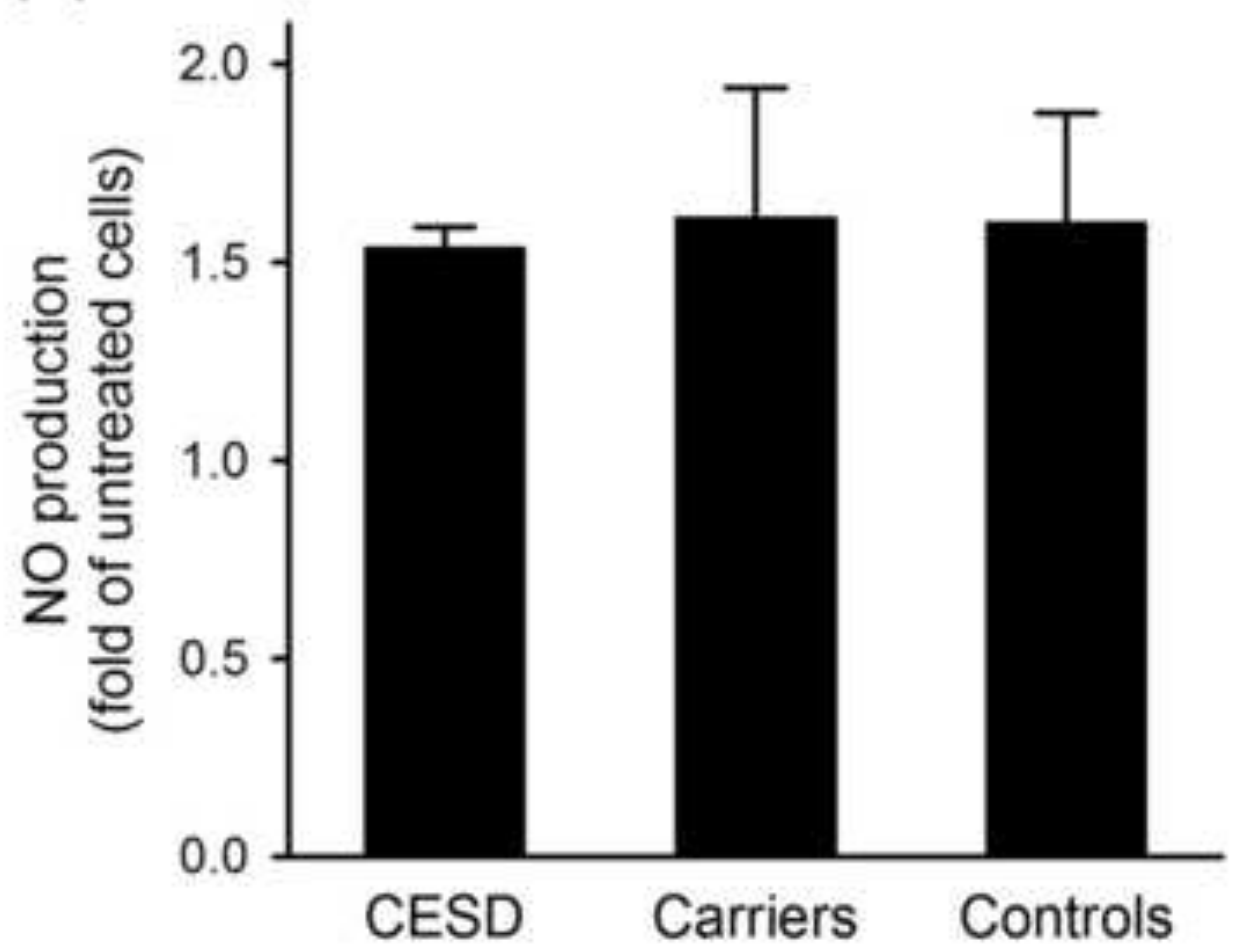

